\title{
Theoretical modeling and experimental study of sodium oleate properties for wastewater cleaning with magnetic nanoparticles stabilized with oleate
}

\author{
Cezarina Morosanu ${ }^{1}$, Larisa Popescu-Lipan ${ }^{1}$, Liviu Sacarescu², Andreea-Roxana Fanaru ${ }^{1}$, and Dorina Creanga ${ }^{1}$ \\ 1"Alexandru Ioan Cuza" University, Physics Faculty, 11 Carol I Avenue, 700506, Iasi, Romania \\ 2"Petru Poni" Institute of Macromolecular Chemistry, Iasi, Romania
}

\begin{abstract}
We present some preliminary results regarding possible reducing of environment phenol contamination with nanotechnology involving. Magnetic nanoparticles stabilized in water dispersion with oleate were prepared for experimental testing of their interaction with wastewater samples containing phenol residues from industrial and sanitation sources. Magnetite with moderate level of cobalt doping was synthesized by co-precipitation method in the form of nanoparticles; further their surface was modified by the reaction with sodium oleate solution. Quantum chemical simulation of oleate structure recommended it for the interaction with water dipolar molecules as well as with metal cations at the nanocores surface. Transmission Electron Microscopy confirmed fine granulation of the prepared sample meaning significant total surface of nanoparticle sample. Phenol loaded water model was let to interact with magnetic nanoparticles in various reaction conditions. Phenol absorbance in the main spectral band showed the concentration diminution following magnetic nanoparticle action in the presence of ultraviolet radiation and hydrogen peroxide supply. Further experiments are planned for the better optimization of the phenol concentration decreasing in the large volumes of wastewater with adequate nanostructures, able to develop efficient interaction mechanisms with pollutant molecules.
\end{abstract}

\section{Introduction}

Cobalt ferrite magnetic nanoparticles have been widely studied in recent decades, due to various technical and biomedical uses, based on the advantages of magnetic control and the large value of surface to volume ratio [1].

The adjustment of the cobalt doping of iron oxide nanoparticles have increased the interest of researchers for the possibility to modulate various properties for possible applications in the life sciences [2,3].

It has been shown that it is possible to use some magnetic nanoparticles to improve wastewater quality by removing chemical pollutants such as heavy metals, phenolic compounds $[4,5]$, drug residues or industrial dyes [6-8].

Some researchers [9] suggest the use of $\mathrm{CoFe}_{2} \mathrm{O}_{4}$ nanoparticles (maximum level of cobalt doping) for the successful treatment of metal-rich industrial effluents. Increasing emphasis is placed on the issue of widely used chemical reagents in many industrial processes most often represented - as a study model - phenol, which is widely used in industry, including in the synthesis of resins, dyes, pharmaceuticals. and pesticides, so that the annual production of phenol exceeds 3 million tons [10]. We performed some preliminary tests focused on the phenol interaction with magnetic nanoparticles stabilized with sodium oleate.

\section{Experimental}

Magnetite nanoparticles with medium cobalt doping level, stabilized with sodium oleate, (notation $\mathrm{Co}_{0,5} \mathrm{Fe}_{2,5} \mathrm{O}_{4}$ ) were synthesized by chemical coprecipitation adapted method [11] and stabilized with sodium oleate [12], while phenolic water samples were adjusted with $1 \mathrm{mM}$ concentration for the interaction with $1 \mathrm{~g} / \mathrm{l}$ cobalt ferrite nanoparticles sample - as suggested by our previous tests.

The characterization of the granulatity of nanoparticles stabilized in water by means of oleate was performed by TEM micrographs (Transmission Electron Microscopy) using Hitachi High-Tech HT7700 device with $0.20-\mathrm{nm}$ resolution. The interaction experiments focused on magnetic nanoparticlle impact on phenolic water were carried aut at room temperature under continuous stirring.

The physicochemical properties and some parameters QSAR (Quantitative Structure Activity Relationship) of the oleate were studied by quantum chemical modeling with Hartree Fock 3-21 G method vy specialized software Spartan 14.

Exposure to UV radiation of phenol containing water was performed using a low pressure discharge tube in mercury vapor (from Philips), with $0.87 \mathrm{~m}$ length, which 
emits UV-C radiation with a maximum of $253,7 \mathrm{~nm}$ (from the mercury spectrum. The total emission power was $30 \mathrm{~W} .10 \mathrm{ml}$ of phenolic water sample in an open glass vessel with diameter of $4 \mathrm{~cm}$ was put at $20 \mathrm{~cm}$ below the center of the discharge tube. The recording of light extinction in phenolic water samples (with the Shimadzu PharmaSpec 1700 spectrophotometer (equipped with quartz vats and specialized data recording and processing software) was performed at different times, between 0 and 120 minutes, from the beginning of the studied interactions. The nanoparticles were removed from the reaction medium by magnetic decantation before the absorbance of the sample was recorded. The rate of decrease in phenol concentration was assessed according to the Beer-Lambert law (the concentration of the absorbing molecule, phenol in this case, is directly proportional to the light absorbance in the sample at a wavelength of $270 \mathrm{~nm}$ relative to a refernce sample).

\section{Results and discussion}

Computational approach has led to dipole moment value of $42.89 \mathrm{D}$ (Table 1) for the sodium oleate -optimized isolated molecule structure and 45.40 D for the hydrated oleate ion; electric dipole vector is orientated from the carboxylic edges toward the opposite molecule/ion terminal as shown in Fig. 1.

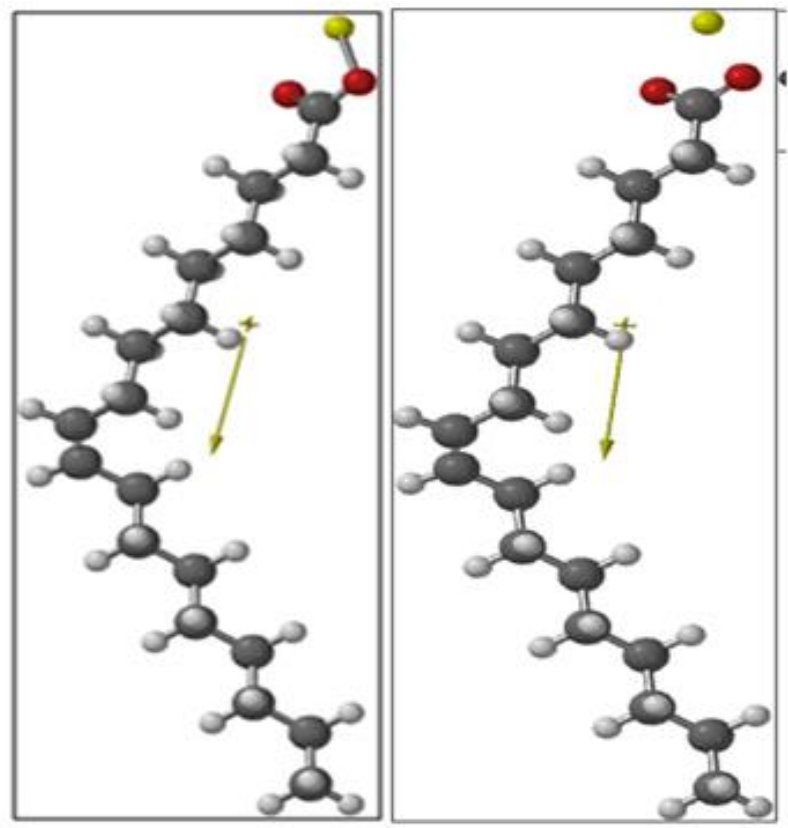

Fig. 1. Equilibrium geometry at ground state for sodium oleate and hydrated oleate ion.

Ionization potential ( $\mathrm{IP}=-\mathrm{E}_{\mathrm{HOMO}}$ according to Koopman's theorem [13]) which is assimilated with the energy of the ground state (HOMO-Highest Occupied Molecular Orbital) has very close values of $-9.11 \mathrm{eV}$ and respectively $-9.23 \mathrm{eV}$ for the hydrated oleate ion.

This means quite possible dipolar interactions with surrounding water dipolar molecules (about 1.9 D) as well as electric interaction with metal cations at the magnetic nanocre surface. The electrostatic potential map (Fig. 2) exhibited some differences that shows that for the hydrated oleate ion (Fig. 2b) the electrostatic potential distribution shows higher values (color turned from blue to green) at the carboxylate edge $\left(\mathrm{COO}^{-}\right)$ compared to molecular sodium oleate (Fig. 2a). This could be taken as an indication of the higher reactivity with neighbouring atoms or molecules of the $\mathrm{COO}$ terminal. The electron affinity approximately equal to the energy ELUMO (LUMO-Lowest Unoccupied Molecular Orbital) has quite different values for the two structures suggesting also higher reactivity of the oleate ion $(0.13 \mathrm{eV}$ for sodium oleate and $5.33 \mathrm{eV}$ for oleate ion).
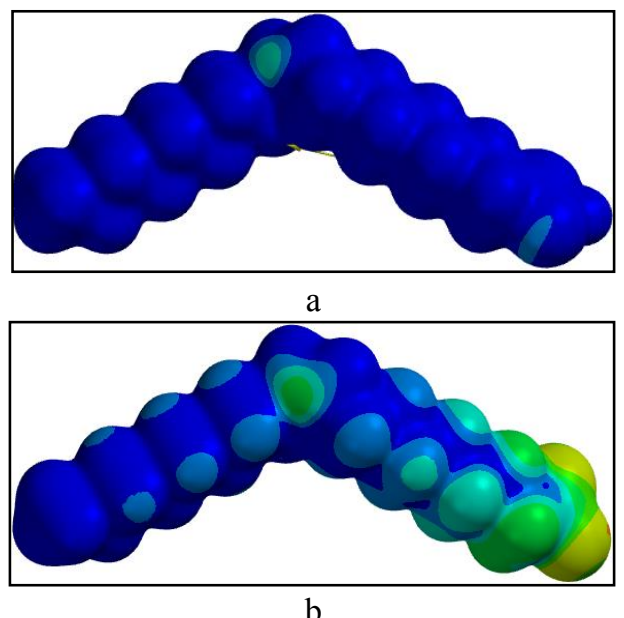

Fig. 2. a - Electrostatic potential map of sodium oleate, $\mathrm{b}$ - Electrostatic potential map of the hydrated oleate ion..

The choice of sodium oleate is motivated by the fact that oleate ions attach strongly to metal ions on the surface of nanoparticles through the carboxylic terminal group (as shown by the use of oleic acid in the stabilization of nanoparticles in non-polar ferrofluids [14-16]. Dispersion in water of manetic nanoparticles stabilized with oleate is ensured by the double-layer organization of oleate ions so that the carboxylic groups in the second layer arrange in contact with dipolar water molecules [12] and give the desired hydrophilicity to the formed colloidal nanoparticles. The results of the analysis of the granularity of the synthesized samples, performed by visualization and dimensional measurement performed by means of TEM micrographs showed average diameters of about $21 \mathrm{~nm}$ with quasispherical or cubic shapes (Fig. 3).

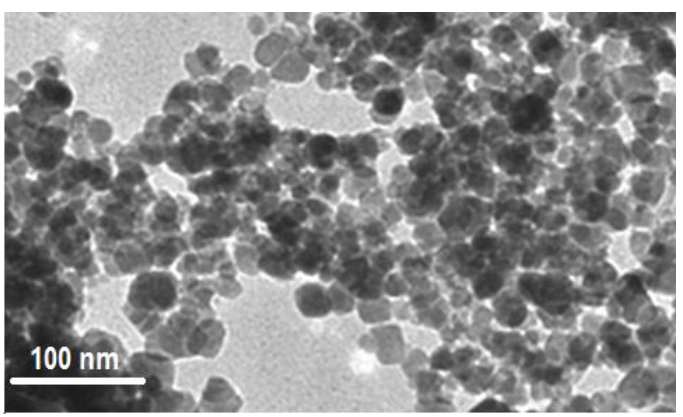

Fig. 3. TEM vizualization of magnetic nanoparticles. 
This is concordant with other published results [17]. Under the experimental conditions detailed above, the first results, with the testing of cobalt ferrite $\mathrm{Co}_{0,5} \mathrm{Fe}_{2,5} \mathrm{O}_{4}$ (magnetite with medium cobalt doping) which were obtained at the relatively high concentration of phenol in water, of $1 \mathrm{mM}$, are presented below.

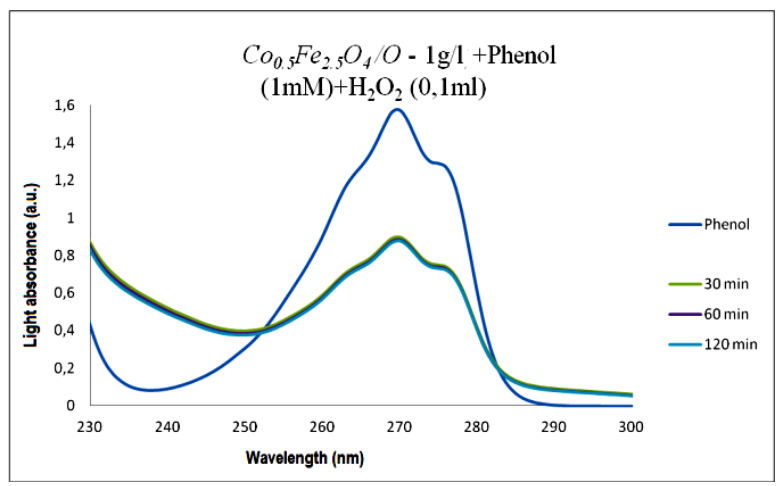

Fig. 4. Electronic absorption spectra of phenol in the presence of $0.1 \mathrm{ml}$ hydrogen peroxide in $10 \mathrm{ml}$ phenolic solution and with $1 \mathrm{~g} / 1$ magnetic nanoparticles.

The result of the action of $1 \mathrm{~g} / \mathrm{l}$ of particles of $\mathrm{Co}_{0,5} \mathrm{Fe}_{2,5} \mathrm{O}_{4}$ stabilized with oleate on phenolic waters (1 $\mathrm{mM}$ ) under hydrogen peroxide supplementation was revealed. After the first 30 minutes of intense stirring, the intensity of the phenol band was found to be reduced by about $50 \%$ at the $270 \mathrm{~nm}$ wavelength - and this change is maintained for up to 120 minutes of continuous interaction (and even up to 180 minutes data not shown here). Addition of hydrogen peroxide $\left(\mathrm{H}_{2} \mathrm{O}_{2}\right)$ does not appear to have changed - neither quantitatively nor qualitatively, the spectrum which implies that the release of highly reactive hydroxyl (HO) radicals did not lead to a change in phenol absorbance under the experimental conditions tested, where the concentration of hydrogen peroxide is relatively small.

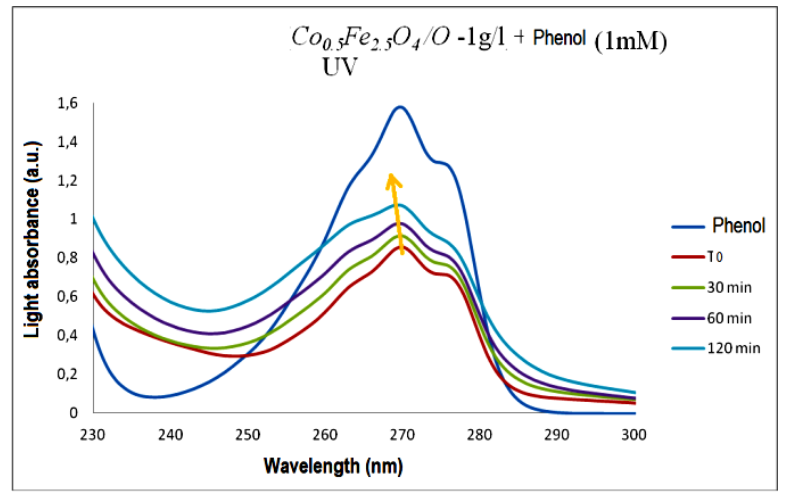

Fig. 5. Influence of UV radiation exposure on the action of $1 \mathrm{~g}$ / 1 oleate-stabilized $\mathrm{Co} 0,5 \mathrm{Fe}_{2,5} \mathrm{O}_{4}$ nanoparticles on phenolic water $(1 \mathrm{mM})$.

The influence of UV radiation may consist in the direct photolysis of phenol molecules with the generation of photolytic products that absorb in the same spectral range but with a shift to blue of the maxima of the main band (Fig. 5, yellow arrow).

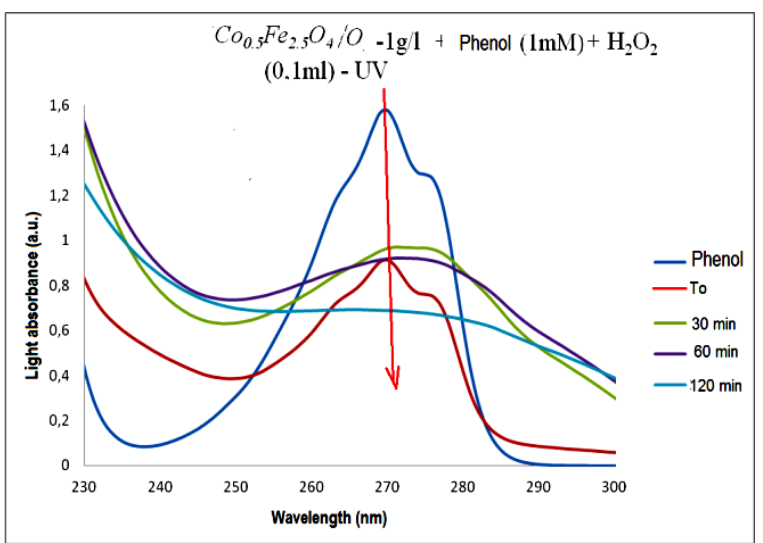

Fig. 6. Influence of hydrogen peroxide on the action of $1 \mathrm{~g} / 1$ of oleate stabilized $\mathrm{Co}_{0,5} \mathrm{Fe}_{2,5} \mathrm{O}_{4}$ nanoparticles on phenolic waters $(1 \mathrm{mM})$ under conditions of exposure to UV radiation.

But especially the photolysis of water - the dominant molecules in the analyzed sample, can generate increased concentrations of hydroxyl radicals that considerably attack phenol (through advanced oxidation processes) leading to hydroquinone or catechol [18] whose presence is reflected in the gradual shift to smaller wavelengths and the progressive increase in intensity in the recorded spectra.The addition of hydrogen peroxide, which, being unstable, releases the reactive hydroxyl $\mathrm{HO}$, can make a significant contribution to the further modification of these products generated by UV radiation (hydroquinone can oxidize and convert to benzoquinone) - as UV radiation would probably do if it would be convenient to considerably increase the exposure time.

Table 1. Quantum chemical modelling results.

\begin{tabular}{|c|c|c|}
\hline Property & $\begin{array}{c}\text { Sodium oleate, } \\
\mathbf{C}_{\mathbf{1 8}} \mathbf{H}_{\mathbf{3} 3} \mathbf{O}_{\mathbf{2}} \mathbf{N a}\end{array}$ & $\begin{array}{c}\text { Oleate ion, } \\
\mathbf{C}_{\mathbf{1 8}} \mathbf{H}_{\mathbf{3} 3 \mathbf{O}} \mathbf{O}_{\mathbf{2}}, \mathbf{\text { in }} \\
\text { water }\end{array}$ \\
\hline Weight & $304.450 \mathrm{amu}$ & $281.460 \mathrm{amu}$ \\
\hline Energy & $-1006.81304 \mathrm{au}$ & $-845.967 \mathrm{au}$ \\
\hline E HOMO & $-9.11 \mathrm{eV}$ & $-9.23 \mathrm{eV}$ \\
\hline E LuMO & $0.13 \mathrm{eV}$ & $5.33 \mathrm{eV}$ \\
\hline Dipole moment & $5.17 \mathrm{D}$ & $45.40 \mathrm{D}$ \\
\hline Area & $407.61 \AA^{2}$ & $396.30 \AA^{2}$ \\
\hline Volume & $356.45 \AA^{3}$ & $348.61 \AA^{3}$ \\
\hline Polarizability & $68.11 \AA^{3}$ & $66.24 \AA^{3}$ \\
\hline
\end{tabular}

Consequently, the absorption spectrum continues to change qualitatively (the two peaks of the band maximum are smoothed) - a fact observed after 30 minutes of reaction, then - quantitatively, the intensity of absorption decreases barely perceptibly at 60 minutes but by almost $25 \%$ compared to the result of the nanoparticles action) after 120 minutes (Fig. 6, red arrow).

\section{Conclusion}

We can assume that the main interaction mechanisms affecting phenol concentration consist in: (i) the adsorption of phenol on the surface of oleate-stabilized nanoparticles, (ii) advanced oxidation of phenol occurs 
under the action of ultraviolet radiation - through radicals from water photolysis - with formation of new products that absorb in the same spectral range.

In the future research project step we shall test more other combinations of reaction parameters to get to the enhanced efficacy of magnetic nanoparticles action on the recalcitrant phenol from wastewaters threatening the environment health and the people life safety.

Funding This research was supported by Romanian project POCU/380/6/13/123623 "PhD students and postdoctoral researchers trained for labor market."

\section{References}

1. S.Y. Srinivasan, K.M. Paknikar, D. Bodas, V. Gajbhiye, Nanomed, 13, 1221 (2018)

2. E. Wetterskog, C.W. Tai, J. Grins, L. Bergström, G. Salazar-Alvarez, ACS Nano, 7, 7132 (2013)

3. S. Chakrabarti, S.K. Mandal, S. Chaudhuri, Nanotechnol., 16, 506 (2005)

4. W. Wang, Y. Liu, T. Li, M. Zhou, Chem. Eng. J., 242, 1 (2014)

5. C. Nadejde, M. Neamţu, R.J. Schneider, V.D. Hodoroaba, G. Ababei, U. Panne, Appl. Surf. Sci., 352, 42 (2015)

6. Y.M. Li, X. Miao, Z.G. Wei, J. Cui, S.Y. Li, R.M. Han, Y. Zhang, W. Wei, Digest J. Nanomater. Biostruct., 11, 1045 (2016)

7. K.K. Singh, K.K. Senapati, K.C. Sarm, J. Environ. Chem. Eng., 5, 2214 (2017)

8. H. Sarvari, E.K. Goharshadi, S. Samiee, N. Ashraf, Phys. Chem. Res., 6, 433 (2018)

9. V. Srivastava, T. Kohout, M. Sillanpää, J. Environ. Chem. Eng., 4, 2922, (2016)

10. L.Y. Taketa, F. Ignachewski, J.C. Villalba, F.J. Anaissi, S.T. Fujiwara, Environ. Sci. Pollut. Res., 22, 894 (2015)

11. R. Massart, IEEE Trans. Magn., 17, 1247 (1981)

12. E. Puscasu, L. Sacarescu, A. Domocos, C. Leoștean, R. Turcu, D. Creanga, M. Balasoiu, Rom. J. Phys., 61, 946 (2016)

13. T. Koopmans, Physica, 1, 104 (1934)

14. R.P. Araújo-Neto, E.L. Silva-Freitas, J.F. Carvalho, T.R.F. Ponte, K.L. Silva, I.H.M. Damasceno, E.S.T. Egito, A.L. Dantas, M.A. Morales, A.S. Carriço, J. Magn. Magn. Mater., 364, 72 (2014)

15. H. Roth, S. Schwaminger, P.F. García, J. Ritscher, S. Berensmeier, J. Nanopart. Res., 18, 99 (2016)

16. K.S. Sharma, R.S. Ningthoujam, A.K. Dubey, A. Chattopadhyay, S. Phapale, R.R. Juluri, S. Mukherjee, R. Tewari, N.G. Shetake, B.N. Pandey, R.K. Vatsa, Sci. Rep., 8, 14766 (2018)

17. L. Popescu, D. Buzatu, M. Balasoiu, C. Stan, B.S. Vasile, L. Sacarescu, D. Creanga, I.O. Ivankov, D. Soloviov, A.-M. Balasoiu-Gaina, Rom. J. Phys., 64, 818 (2019)

18. A. Maleki, A.H. Mahvi, M. Alimohamadi, A. Ghasri, Pak. J. Biol. Sci., 9, 2338 (2006). 